\title{
BAYESIAN CORRECTION FOR MEASUREMENT ERROR FOLLOWING GROUP-BASED EXPOSURE ASSESSMENT IN A CASE-REFERENT STUDY
}

Igor Burstyn, ${ }^{1}$ Frank de Vocht, ${ }^{2}$ Hyang-Mi Kim, ${ }^{3}$ Nicola Cherry ${ }^{4}$ Drexel University, Philadelphia, USA; ${ }^{2}$ University of Manchester, Manchester, UK; ${ }^{3}$ University of Calgary, Calgary, Canada; ${ }^{4}$ University of Alberta, Edmonton, Canada

10.1136/oemed-2011-100382.145

Objectives We applied Bayesian analysis to case-referent data on occupational noise exposure and death from ischaemic heart disease (IHD) and contrast analyses with and without correction for measurement error in group-level noise exposure estimates.

Methods A 1:1 matched case-referent study nested in an industrial cohort in England resulted in 117 matched sets; 7225 area noise measurements in $\mathrm{dBA}$ from 215 buildings were the basis of modeling building-specific average exposures during the decade of in service IHD death. An additive quasi-Berkson error model was assumed. Bayesian analysis was conducted under varying assumptions about magnitude of error (with SD of error (SDe) up to $10 \mathrm{dBA}$ ) and a prior strength of hypothesis (flat vs informative -- $98 \%$ range $(1.00,1.02)$-- prior on OR). All analyses ignored matching and were conducted without adjustment for confounders to estimate $\log (\mathrm{OR}) / \mathrm{dBA}$ in a logistic disease model.

Results Analysis not corrected for measurement error with flat prior yielded OR 0.99 (95\% CrI 0.96-1.02). With flat prior on OR with measurement error correction, OR had 95\% CrI 0.97-1.02; with informative prior on the association, the corresponding $\mathrm{OR}$ is $1.01,95 \% \mathrm{CrI} 1.00-1.02$, same as prior. The posterior distribution of SDe had median 1.6 (95\% CrI 1.2-2.0) dBA.

Conclusions Measurement error did not bias the uncorrected results. Conditional logistic regression with adjustment for confounders is congruent with Bayesian analysis (115 pairs, OR $0.98,95 \%$ CI 0.94 to 1.02 ). Analysis provided insights into plausible magnitudes of measurement error in the study. Extension of this methodology to consider matching, confounders and retrospective nature of data is required. 